\title{
Online-Planspiele als Wegbereiter für internationale und digitale Hochschullehre
}

\author{
Sven Ivens und Konstantin Kaiser
}

\section{Zusammenfassung}

Dieser Beitrag untersucht, wie die innovative Methode des Online-Planspiels die Hochschullehre in Deutschland digitaler, kooperativer und internationaler gestalten kann. Ein solches Online-Planspiel hat Studierende der Politikwissenschaft an den Universitäten Antwerpen, Göttingen und Krakau im Wintersemester 2019/2020 auf einer Weboberfläche digital verhandeln und interagieren lassen. Die Erfahrungen, die die Studierenden während des Online-Planspiels gemacht haben, bilden die Grundlage dieser Untersuchung. Dabei wurde folgenden Fragen nachgegangen, um die Ergebnisse in den größeren Kontext der Hochschullehre einzuordnen: Wie bewerten die Studierenden das Online-Planspiel? Wie haben sie das digitale und internationale Arbeitsumfeld wahrgenommen? Welche Fähigkeiten fördert die Teilnahme an einem OnlinePlanspiel? Beantwortet wurden diese Fragen einerseits in Selbstreflexionen, andererseits in standardisierten Interviews, deren Inhalte mit einer evaluativen qualitativen Inhaltsanalyse analysiert wurden. Das Online-Planspiel wird sehr positiv bewertet, fast alle Teilnehmenden berichten von einem Lerneffekt. Es werden wichtige Lektionen für Online-Planspiele generell hinsichtlich des Workloads, des Durchführungszeitpunkts, des Umgangs mit Drop-outs und der digitalen Kommunikation aufgezeigt und Überlegungen angestellt, wie diese neuartige Lehrmethode dahin gehend zukünftig verbessert werden kann.

\footnotetext{
S. Ivens $(\bowtie)$

Georg-August-Universität Göttingen, Göttingen, Deutschland

E-Mail: sven.ivens@uni-goettingen.de

K. Kaiser

planpolitik GbR, Berlin, Deutschland
} 


\section{Schlüsselwörter}

Hochschulkooperation • Internationalisierung • Online-Planspiele • Digitale Planspiele • Digitalisierung • Online-Lernformate • Digitale Hochschullehre • Politikwissenschaft

\section{$1 \quad$ Einführung}

Das universitäre Curriculum steht vor drei großen Herausforderungen: Zum einen sollte in einer grenzenlosen und globalisierten Welt auch die Hochschullehre international sein. Doch schon vor der COVID-19-Pandemie war der ERASMUSAustausch ein Höhepunkt des Studiums und kein Normalfall, da Reise- und Organisationskosten für viele Studierende zu hoch sind. Die zweite Herausforderung ist der Präsenzcharakter der Universität. Nur ein Bruchteil der Seminare findet in einer digitalen Umgebung statt. Damit kapselt sich der universitäre Alltag von der Lebenswelt des 21. Jahrhunderts ab. Längst gibt es reine OnlineUniversitäten, die immer enger mit Karriereportalen zusammenarbeiten und damit die Berufsrelevanz eines Hochschulabschlusses langfristig infrage stellen. Die dritte Herausforderung ist zugleich auch die bekannteste: überfüllte Seminarräume und Vorlesungen, in denen wenig abwechslungsreiche Frontallehre praktiziert wird. Wie kann Hochschullehre diesen Herausforderungen begegnen?

Dieser Artikel geht der Frage nach, inwieweit eine digitale Lernumgebung in Form eines Online-Planspiels sowohl die internationale Kooperation zwischen Universitäten als auch die Interaktion von Studierenden fördern kann. Dieser Artikel beschreibt auch, wo das Format Schwachstellen zeigt, und identifiziert Verbesserungspotenziale. Die empirische Grundlage der Untersuchung ist ein Seminar, welches als internationales Online-Planspiel über Lobbyismus in der EU über zwei Wochen auf einer Webspieloberfläche vollständig online durchgeführt wurde.

Um herauszufinden, wie nützlich eine solche interaktive, digitale und internationale Methode ist, wurden Selbstreflexionsaufgaben der Studierenden und fünf leitfragengestützte Interviews mit Teilnehmenden zusammen mit einer evaluativen qualitativen Inhaltsanalyse nach Kuckartz (2018) analysiert.

Das Online-Planspiel wurde dabei positiv bewertet, fast alle Teilnehmenden geben an, mehr über die Europäische Union gelernt zu haben, und berichten von einer Verbesserung ihrer praktischen Fähigkeiten im Bereich des professionellen Schreibens (zum Beispiel Presseberichte und Policy Paper) sowie ihres Verhandlungsgeschicks. Es gab aber auch konstruktive Kritik. Einerseits wurde 
die entstehende Flexibilität und Autonomie der digitalen Arbeitsumgebung gelobt, andererseits fehlten den Teilnehmenden Face-to-Face-Diskussionen. Späte oder fehlende Reaktionen anderer Mitspielender werden besonders negativ wahrgenommen. Auch ist der Arbeitsaufwand des Online-Planspiels für Studierende und Dozierende höher als vorher angenommen. Insgesamt konnten viele wichtige Lektionen über das Online-Planspiel als innovatives Lernformat in dieser ersten Durchführung und Analyse gewonnen werden.

In dem Beitrag wird zuerst in die wissenschaftliche Diskussion über OnlinePlanspiele in der Hochschulbildung eingeführt, um Ziele und Forschungsfragen zu identifizieren. Auf dieser Basis wird in einem zweiten Schritt das durchgeführte Online-Planspielseminar vorgestellt und analysiert. Die Ergebnisse der Evaluation und die daraus gewonnenen Erkenntnisse für den Einsatz von Online-Planspielen werden im Fazit reflektiert.

\section{Wenig Forschung, aber hohe Erwartungen: Online-Planspiele in der (politikwissenschaftlichen) Hochschullehre}

Analoge Planspiele sind in der politikwissenschaftlichen Bildung anerkannt, oft benutzt und laufend erforscht (siehe Petrik und Rappenglück 2017). Empirische Studien zeigen die positive Wirkung von Planspielen im universitären Bereich (Fink 2015; Lohmann 2019; Duchatelet 2019). Gerade im Bereich der Politikwissenschaften setzten sich Hochschullehrer*innen mit der Planspielmethode auseinander (siehe unter anderem Meßner et al. 2018; Mungo 2020), aber auch in anderen Bereichen, wie dem Bereich Bildung für Nachhaltige Entwicklung oder in den Wirtschaftswissenschaften (Snyder 2003; Ivens et al. 2020). Analoge Planspiele werden in der Form von Model United Nations oder der EuroSim (Jones und Bursen 2018) schon als fächer- und länderübergreifende Unterrichtsmethode in der Hochschulbildung eingesetzt. Planspiele folgen generell einem Active-Learning-Ansatz (Jones und Bursens 2018), welcher die Förderung von Problemlösungsfähigkeit, einen nachhaltigen Wissenserwerb und ein positives Lernumfeld vorsieht (Snyder 2003).

Im Vergleich zu analogen Planspielen wurden der Einsatz und die Wirkung von Planspielen in einer digitalen Umgebung bisher nur wenig empirisch erforscht (Bachen et al. 2015), umso höher sind jedoch die Erwartungen. Generell gibt es drei grundlegende Merkmale von Online-Planspielen, sie können entweder zeitlich versetzt (asynchron) oder gleichzeitig (synchron) durchgeführt werden, es 
ist möglich, sie komplett online oder in einem Blended-Learning-Format stattfinden zu lassen, und die Teilnehmenden können vollständig anonym partizipieren (Wills et al. 2011). Besonders asynchrone Online-Planspiele eignen sich für die Hochschullehre, da diese über einen längeren Zeitraum durchgeführt werden und dadurch den Teilnehmenden mehr Autonomie und Flexibilität einräumen, was wiederum intensive Recherche, inhaltlich gehaltvolle Verhandlungen und eine höhere Rollenidentifikation ermöglicht (Wills et al. 2011; Kaiser et al. 2017). Zudem wird angenommen, dass die intrinsische Motivation der Teilnehmenden (Le et al. 2013) sowie Verhandlungsfähigkeiten, Offenheit und Teamwork (Gabriel 2012) gestärkt werden. Die Herausgeber*innen von Online-Planspielen sehen in ihren Produkten viel Potenzial für den Online-Bildungsbereich, eine Vervielfachung der Reichweite, den Vorteil einer ortsungebundenen Durchführung (Kaiser et al. 2017) und die kostengünstige Möglichkeit eines Austauschs zwischen Teilnehmenden aus verschiedenen Ländern (Raiser et al. 2018). Die Anonymität von Online-Planspielen kann außerdem ein sicheres Umfeld für die Teilnehmenden erzeugen, in welchem sie ohne Druck neue Ideen frei äußern und vorurteilsfrei an dem Planspiel teilnehmen können (Lybeck et al. 2010; Wills et al. 2011).

Auch mit Blick auf die politikwissenschaftliche Hochschullehre herrscht eine Forschungslücke bezüglich der Erforschung von Einsätzen digitaler und onlinebasierter Planspiele in der universitären Bildung. Eine Metastudie über den Einsatz digitaler Spiele, einschließlich digitaler Rollen- und Planspiele, in der Hochschullehre über die Jahre 2010 bis 2016 konnte nur vier Studien aus dem Bereich Politikwissenschaft ausfindig machen, im Gegensatz zu Ingenieurwissenschaft mit acht oder Management mit 21 Studien (Vlachopoulos und Makri 2017). Ein positives Beispiel ist die Studie von Schnurr et al. (2013). Aus der Analyse des Feedbacks der Teilnehmenden eines Blended-Learning-Planspiels über internationale Klimaverhandlungen ergab sich, dass besonders die Skills im Bereich der Policy-Analyse, des Verhandlungsgeschicks und des Koalitionsbildens durch die Simulation gestärkt wurden. Gerade die onlinegestützten Diskussionen waren für das Lernen förderlich und in die digitale Kommunikation konnten sich die eher stillen Studierenden besonders einbringen. Die Autor*innen warnen jedoch zum einen davor, dass das Planspiel mehr Aufwand für die Studierenden bedeutete als vorher angenommen. Zum anderen schätzten die Studierenden den Face-toFace-Kontakt untereinander und organisierten dafür private Treffen (Schnurr et al. 2013). Neben diesem Beispiel kam es noch zu einigen wenigen anderen Einsätzen von Planspielen mit digitalen Elementen in der politikwissenschaftlichen Hochschulbildung (Naidu et al. 2000; Horn et al. 2016), und mit der EuroSim gibt es ein gutes Beispiel für ein internationales analoges Planspiel (Jones und Bursens 2018), jedoch keines, das den Vorteil der internationalen Kooperation 
zwischen den Fachbereichen verschiedener Universitäten über ein digitales Planspiel nutzt. Dies ist sowohl in der hochschuldidaktischen Forschung als auch bei den Internationalisierungsstrategien der Fachbereiche und Universitäten eine Lücke.

Schaut man sich generelle Online- und Blended-Learning-Formate an, bieten diese verschiedene Vorteile und Risiken. Nach Deschant und Goeman (2015) erhöhen diese Formate zwar die Leistung der Teilnehmenden, gehen dabei aber mit einer erhöhten Drop-out-Rate einher. Auch zeigt sich in einer neuen Metastudie, dass der Einsatz moderner Technologie, wie Digitalisierung oder Virtual Reality, den positiven Effekt simulationsbasierten Lernens in der Hochschulbildung erhöht (Chernikova et al. 2020).

Online-Lehrformate fördern die Unabhängigkeit der Teilnehmenden und schaffen Freiräume, dafür können sich mangelnde Face-to-Face-Interaktion und die verringerte persönliche Betreuung negativ auf die Motivation der Teilnehmenden und so das Risiko eines Kursabbruchs auswirken (Cho und Jnassen 2009; Fryer und Bovee 2016). Vanslambrouck und Kolleg*innen (2016) haben dafür Studierende zu ihren Erfahrungen mit Online- und Blended-Learning-Umgebungen befragt und festgestellt, dass Studierende zwar die freie Arbeitseinteilung sehr schätzen, dafür den fehlenden persönlichen Kontakt als negativ beurteilen, da dieser zu einer gewissen Distanz bei allen Beteiligten führt. In einer Studie wird zu Studierendenmotivation und Online- und Blended-Learning-Formaten die Praxisempfehlung gegeben, Teilnehmenden vorher genau klarzumachen, wie hoch der Arbeitsaufwand des Lernformates ist, da ein unerwarteter und hoher Arbeitsaufwand, welcher durch Nachtarbeit kompensiert werden muss, oft ein Grund ist, warum Studierende Online- und Blended-Learning-Formate negativ beurteilen (Vanslambrouck et al. 2018).

Anhand des Forschungsstandes kann man von einem Online-Planspiel in der Politikwissenschaft erwarten, dass es die intrinsische Motivation stärkt, Autonomie und digitales Arbeiten näherbringt und verschiedene Skills, wie zum Beispiel das Verhandlungsgeschick, stärken sollte. Auf der anderen Seite birgt es Risiken: Da die gesamte Kommunikation online stattfindet, könnte der frustrationsbedingte Drop-out steigen, auch die Arbeitsbelastung könnte höher werden als zuvor angenommen. 


\section{$3 \quad$ Analyse des Online-Planspielseminars}

\subsection{Ziele und Forschungsfragen der Begleitstudie}

Drei Ziele wurden für die Forschung zum Online-Planspielseminar festgelegt, aus denen sich gezielte Forschungsfragen ergeben. Erstens sollen generell Verbesserungsmöglichkeiten für Online-Planspiele und Planspielseminare identifiziert werden. Zweitens sollen der Lerneffekt eines Online-Planspiels und dessen Wirkung auf die Studierenden exemplarisch erforscht werden. Und drittens (aber nicht zuletzt) soll offengelegt werden, welche Anwendungsmöglichkeiten OnlinePlanspiele in der digitalen Hochschullehre haben können. Aus den Zielen ergeben sich folgende drei Forschungsfragen:

1. Wie bewerten die Studierenden das Online-Planspiel, die Online-Plattform und den Arbeitsaufwand für das Online-Planspielseminar?

2. Wie empfinden die Studierenden den Lerneffekt, die Internationalisierung, das digitale Arbeitsumfeld und den Wirklichkeitsgehalt der Simulation?

3. Erwerben oder verbessern die Studierenden durch die Teilnahme am OnlinePlanspiel berufs- und studienrelevante Fähigkeiten? Wenn ja, welche?

Diese Forschungsfragen werden in der qualitativen Analyse der Selbstreflexionen und in Interviewaussagen der teilnehmenden Studierenden beantwortet, der genaue Vorgang der Analyse wird im Kapitel zum Studiendesign beschrieben.

\subsection{Das Online-Planspiel „Lobbying in the EU“}

Das untersuchte Online-Planspiel „Lobbying in the EU“ simuliert den Gesetzgebungsprozess in der Europäischen Kommission und in einem Ausschuss des Europäischen Parlamentes. Das Spiel wurde vom Anbieter planpolitik für den politikwissenschaftlichen Einsatz an Universitäten entwickelt und basiert technisch auf der Planspiel-Plattform Senaryon (www.senaryon.de). Beispiele der Benutzeroberfläche des Online-Planspiels finden sich im digitalen Appendix. ${ }^{1}$ Die Aufgabe der Teilnehmenden ist es, gemeinsam Maßnahmen zu definieren, mit deren Hilfe die $\mathrm{CO}_{2}$-Emissionen im Verkehrssektor bis 2030 um $40 \%$ verringert werden können. Da die Ziele bewusst ambitioniert und wenig konkret formuliert sind, stehen die Lobbygruppen diesem Vorhaben ambivalent gegenüber. Je nach

\footnotetext{
${ }^{1}$ Siehe: https://bit.ly/31STDwE. Zugegriffen: 26.10.2020.
} 
Inhalt kann das neue EU-Gesetz die Ziele der Industrie- und Umweltgruppen sowohl fördern als auch bremsen. Welche Inhalte es in den Gesetzestext schaffen, hängt davon ab, wie die verschiedenen Gruppen im Spiel miteinander kooperieren. Eine Hälfte der Studierenden übernimmt Akteur*innen der Europäischen Institutionen und erhält die Aufgabe, diese Ziele mit konkreten Inhalten zu füllen und dafür einen Zeitplan zu entwickeln. Die Lobbyist*innen werden von der anderen Hälfte der Studierenden gespielt. Nach einer Woche tauschen die Studierenden ihre Rollen. Die Ergebnisse werden am Ende der ersten Spielwoche erst von der EU-Kommission in einem Verordnungsentwurf festgehalten und danach in der zweiten Woche im EU-Parlamentsausschuss zur Abstimmung gestellt. Bei der Rollenverteilung wurde auf die gleiche Teamgröße von Interessenvertretungen und politischen Gruppierungen geachtet, wobei sich die Größe der Fraktionen im EU-Parlament an der realen Größe orientiert. Um den Charakter der Internationalisierung und Kooperation zu erfüllen, wurde innerhalb dieser Spielgruppen ebenfalls darauf geachtet, dass überall Studierende unterschiedlicher Universitäten vertreten waren. Spielinhalte und Verkehrssprache waren Englisch.

Ein mehrwöchiges Online-Planspiel verfügt nicht über reizvolle gleichzeitige und kürzere Face-to-Face-Interaktionen. Um dennoch eine intrinsische Motivation in den Spielenden zu erzeugen, legt das Spieldesign einen Schwerpunkt auf die wechselseitige Abhängigkeit und hohe Kooperation zwischen den Aktivitäten der Teilnehmenden. Es wird darauf geachtet, dass individuelle Aktionsräume geschaffen werden (zum Beispiel Stellungnahmen veröffentlichen, Gesetzestexte entwickeln und konkretisieren), die sichtbaren Einfluss auf das gesamte Spielergebnis haben, um das Selbstwirksamkeitsgefühl der Teilnehmenden zu erhöhen. So tragen alle Teilnehmenden eine hohe Verantwortung für den gesamten Spielprozess. Damit einher geht das Risiko, dass inaktive Studierende wichtige Aufgaben nicht wahrnehmen und der Spielprozess ins Stocken gerät.

Um für einen geordneten Spielverlauf zu sorgen, bildet das Aufgabensystem den Kern des Spiels. Damit wird das Spiel in mehrtägige Phasen mit verbindlichen Deadlines unterteilt. Die gestellten Aufgaben sind sehr vielfältig und in drei Kategorien unterteilt:

1. Interaktive und kommunikative Gruppenaufgaben (zum Beispiel Erarbeitung einer Strategie im internen Gruppenchat, Austausch über das Nachrichtensystem mit anderen Gruppen).

2. Spielkritische kooperative Gruppenaufgaben, die unter anderem in kollaborativen Textfeldern erarbeitet werden und aufeinander aufbauen (zum Beispiel Phase 1: Entwicklung eines Vorschlags durch Gruppe A, Phase 2: Bewertung dieses Vorschlags durch Gruppe B). 
3. Einzelaufgaben, die einerseits zur Erarbeitung und der Zusammenfassung von Spielinhalten dienen (zum Beispiel Recherche im Internet, Formulierung einer Position), und andererseits Reflexionsaufgaben, in denen beispielweise der Einfluss des eigenen Handelns auf den Spielverlauf bewertet wird. Diese Einzelaufgaben werden in einem Textfeld bearbeitet und dienen den Dozierenden als Grundlage für die Benotung.

Ein weiterer großer Bereich der Software ist für die Teilnehmenden gesperrt und nur für die Dozierenden verfügbar. In diesem Moderationsbereich erfolgt die Kommunikation mit den Teilnehmenden, ihre Aktivitäten können nachverfolgt und die abgeschlossenen Aufgaben bewertet werden.

\subsection{Studiendesign}

Das hier exemplarisch untersuchte Online-Planspiel wurde in der Mitte des Wintersemesters 2019/2020 für zwei Wochen gespielt. Es nahmen insgesamt 22 Studierende der Universitäten Antwerpen, Göttingen und Krakau daran teil, sieben Teilnehmende sind im Laufe des Spiels ausgestiegen, sodass das Spiel von 15 Teilnehmenden beendet wurde. Die Studierenden der Universität Göttingen waren sowohl aus dem Bachelor Politikwissenschaft als auch aus dem Master Euroculture und haben Creditpoints und Noten für die Teilnahme und die Bearbeitung der Aufgaben im Online-Planspiel bekommen. Die Studierenden der Universität Krakau und Antwerpen waren Masterstudierende, haben aber keine Creditpoints bekommen und an dem Projekt lediglich als extracurriculare Aufgabe teilgenommen.

Bei der gestellten Selbstreflexionsaufgabe sollten die Studierenden über das Planspiel selbst sowie die Aktivitäten und Lernerfolge während des Planspiels reflektieren. Insbesondere sollten sie auf die digitale Zusammenarbeit mit Teilnehmenden aus verschiedenen Ländern, den Wirklichkeitsgehalt der Simulation aus ihrer Perspektive sowie generelle Vor- und Nachteile der Online-Simulation eingehen.

Zusätzlich zu den Selbstreflexionen wurden fünf standardisierte Interviews von trainierten studentischen Mitarbeitenden durchgeführt. Die Teilnahme basierte auf Freiwilligkeit. Die Interviews wurden entweder auf Deutsch oder Englisch geführt, je nach Wunsch der Interviewten. Die standardisieren Interviews waren jeweils zwischen 15 bis 20 Minuten lang, wurden innerhalb von zwei 
Wochen nach der Simulation geführt und hatten die Bewertung des OnlinePlanspiels seitens der Studierenden wie auch den Vergleich des Planspiels mit Präsenzseminaren in ihrem Studium zum Thema.

Die Selbstreflexionen und Interviews dienten als Analyseeinheiten und wurden durch eine evaluative qualitative Inhaltsanalyse nach Kuckartz (2018) untersucht, das genaue Vorgehen und die gebildeten Kategorien mit Ankerbeispielen finden sich im digitalen Appendix. ${ }^{2}$

\subsection{Ergebnisse der Evaluation/der Begleitstudie}

\subsubsection{Generelle Bewertung des Online-Planspiels}

Die generelle Bewertung des Online-Planspiels ist sehr gut. Fast $85 \%$ der codierten Aussagen haben eine positive Bewertung (siehe Tab. 1) und es gab insgesamt

Tab. 1 Ergebnisse der qualitativen Inhaltsanalyse zu der Bewertung des Planspiels, dessen Online-Plattform und des Arbeitsaufwandes. (Eigene Darstellung)

\begin{tabular}{|c|l|c|c|c|}
\hline \multirow{2}{*}{ Kategorie } & \multicolumn{1}{c}{ Unterkategorie } & $\begin{array}{c}\text { Nennung } \\
\text { Selbstreflexion }\end{array}$ & $\begin{array}{c}\text { Nennung } \\
\text { Interview }\end{array}$ & $\begin{array}{c}\text { Nennung } \\
\text { Gesamt }\end{array}$ \\
\hline \multirow{2}{*}{$\begin{array}{c}\text { Bewertung } \\
\text { des Planspiels }\end{array}$} & Positive Bewertung & $15(78,8 \%)$ & $7(100 \%)$ & $22(84,6 \%)$ \\
\cline { 2 - 5 } & Gemischte Bewertung & $3(15,9 \%)$ & - & $3(11,5 \%)$ \\
\hline \multirow{2}{*}{$\begin{array}{c}\text { Bewertung } \\
\text { der Online- } \\
\text { Plattform }\end{array}$} & Gemischte Bewertung & $3(20 \%)$ & - & $1(3,9 \%)$ \\
\cline { 2 - 5 } & Negative Bewertung & $3(20 \%)$ & - & $12(63,2 \%)$ \\
\hline \multirow{2}{*}{$\begin{array}{c}\text { Arbeitsauf- } \\
\text { wand des } \\
\text { Planspiels }\end{array}$} & Genaurichtig & $1(5,3 \%)$ & $1(25 \%)$ & $3(15,7 \%)$ \\
\cline { 2 - 5 } & Eher zuhoch & $7(63,6 \%)$ & $1(50 \%)$ & $8(61,5 \%)$ \\
\cline { 2 - 5 } & Zu hoch & $3(27,3 \%)$ & - & $3(23,1 \%)$ \\
\hline
\end{tabular}

${ }^{2}$ Siehe: https://bit.ly/31STDwE. Zugegriffen: 26.10.2020. 
nur eine negative Bewertung. Die gemischten Reaktionen beziehen sich auf den Wechsel der Rollen nach einer Woche und gleichfalls auf inaktive Gruppenpartner*innen. Die positiven Bewertungen umfassten unter anderem die höhere Motivation durch das Online-Planspiel, die Abwechslung im Vergleich zu den regulären Seminaren der Teilnehmenden, das Eintauchen in eine andere Rolle und in die Prozesse der EU sowie den praktischen Ansatz der Online-Simulation.

Die Online-Plattform für das Planspiel wird ebenfalls mehrheitlich positiv bewertet, jedoch gibt es hier mehr gemischte Reaktionen und auch kritische Bewertungen der Plattform (siehe Tab. 1). Die Online-Plattform Senaryon und deren Benutzeroberfläche werden als gut strukturiert, informativ und dynamisch beschrieben. Die gemischten Reaktionen waren prinzipiell positiv, enthielten aber Verbesserungsvorschläge, wie das Einbauen eines generellen Forums, die Möglichkeit, Chatnachrichten als wichtig zu markieren und das Zeichenlimit für Aufgaben aufzuweichen. Als negative Bewertungen wurden vor allem technische Probleme angesprochen.

Der Arbeitsaufwand für das Online-Planspiel wird von den Studierenden als zu hoch wahrgenommen, nur zwei Studierende merkten an, das sie die Herausforderungen und den Stress der Online-Simulation sehr erfrischend fanden (siehe Tab. 1). Dagegen zeigen über $80 \%$ der codierten Aussagen, dass die Teilnehmenden den Workload der Online-Simulation als hoch empfanden. Gerade die Aussagen, die den Arbeitsaufwand als zu hoch beziffern, gehen explizit auf die Creditpoints des Seminars ein und geben an, dass man dieselbe Anzahl an Creditpoints mit bedeutend weniger Arbeit im selben Modul erwerben könne. Das könnte aus Sicht der Studierenden eine abschreckende Wirkung auf die Teilnahme an dem Seminar haben - oder wie es in einer Selbstreflexion ausgedrückt wurde:

„Because why should anybody invest, at least, ten times more time and nerves if they can easily earn the credits with much, much less?“

\subsubsection{Bewertung der (digitalen) internationalen Zusammenarbeit}

Das Online-Planspiel hat aus der Sicht der Teilnehmenden einen sehr positiven Lerneffekt. Insgesamt waren über $90 \%$ aller Aussagen bezüglich des Lerneffektes positiv (siehe Tab. 2). Die Teilnehmenden konnten lernen, wie der Gesetzgebungsprozess der EU funktioniert, wie schwer es ist, politische Maßnahmen im Kontext des Mehrebenensystems der EU zu etablieren, welche Bedeutung Lobbyismus in diesem System hat und wie Lobbyist*innen vorgehen.

Ähnlich positiv wird die Internationalisierung des Online-Seminars wahrgenommen, $90 \%$ der Aussagen sehen das Seminar zumindest etwas internationalisiert, für $65 \%$ ist es sogar ausgeprägt internationalisiert (siehe Tab. 2). Die 
Tab. 2 Ergebnisse der qualitativen Inhaltsanalyse zum Lerneffekt, der wahrgenommenen Internationalisierung, digitalen Arbeitsumwelt und der Wirklichkeitsnähe der OnlineSimulation. (Eigene Darstellung)

\begin{tabular}{|c|c|c|c|c|}
\hline Kategorie & Unterkategorie & $\begin{array}{c}\text { Nennung } \\
\text { Selbstreflexion }\end{array}$ & $\begin{array}{l}\text { Nennung } \\
\text { Interview }\end{array}$ & $\begin{array}{c}\text { Nennung } \\
\text { Gesamt }\end{array}$ \\
\hline \multirow{3}{*}{ Lerneffekt } & Positiver Lerneffekt & $10(58,8 \%)$ & $7(77,8 \%)$ & $17(65,4 \%)$ \\
\hline & Eher positiver Lerneffekt & $6(35,4 \%)$ & $1(11,1 \%)$ & $7(26,9 \%)$ \\
\hline & Kein Lerneffekt & $1(5,8 \%)$ & $1(11,1 \%)$ & $2(7,7 \%)$ \\
\hline \multirow{3}{*}{$\begin{array}{l}\text { Internatio- } \\
\text { nalisierung }\end{array}$} & Ausgeprägt & $9(69,2 \%)$ & $4(57,1 \%)$ & $13(65 \%)$ \\
\hline & Wenig ausgeprägt & $2(15,4 \%)$ & $3(42,9 \%)$ & $5(25 \%)$ \\
\hline & Nicht ausgeprägt & $2(15,4 \%)$ & - & $2(10 \%)$ \\
\hline \multirow{3}{*}{$\begin{array}{c}\text { Wahrnehmung } \\
\text { digitaler } \\
\text { Arbeitsumgebung }\end{array}$} & Positive Wahrnehmung & $3(30 \%)$ & $8(50 \%)$ & $11(42,3 \%)$ \\
\hline & $\begin{array}{l}\text { Gemischte } \\
\text { Wahrnehmung }\end{array}$ & $5(50 \%)$ & $5(31,3 \%)$ & $10(38,5 \%)$ \\
\hline & Negative Wahrnehmung & $2(20 \%)$ & $3(18,7 \%)$ & $5(19,2 \%)$ \\
\hline \multirow{3}{*}{$\begin{array}{c}\text { Wirklichkeitsnähe } \\
\text { der Simulation }\end{array}$} & Ausgeprägt & $2(14,3 \%)$ & - & $2(12,5 \%)$ \\
\hline & Wenigausgeprägt & $9(64,3 \%)$ & $1(50 \%)$ & $10(62,5 \%)$ \\
\hline & Nicht ausgeprägt & $3(21,4 \%)$ & $1(50 \%)$ & $4(25 \%)$ \\
\hline
\end{tabular}

Zusammenarbeit mit Studierenden aus anderen Ländern wird dabei als sehr positiv und motivierend angesehen. Auch im Vergleich zu anderen Seminaren wird die Internationalisierung des Planspielseminars als besonderes Extra wahrgenommen.

Die digitale Arbeitsumgebung hingegen wird sehr unterschiedlich bewertet. Einerseits bewerten etwas mehr als $40 \%$ der Aussagen das Online-Arbeiten als positiv (siehe Tab. 2). So wird vor allem die dadurch entstehende Professionalität, die Selbstständigkeit, Flexibilität und Autonomie sehr geschätzt, die ebenfalls schüchterne Teilnehmende ermutigt, sich in Diskussionen einzubringen, was dieser Interviewausschnitt deutlich macht: 
„I think the discussion went well online. I think it is easier to make your opinions heard online, because when you talk face to face you are sometimes too shy or don't dare to say things, while online you are just typing and it is easier to just say everything you want to say.“

Auf der anderen Seite zeigen fast $20 \%$ der Aussagen eine negative Wahrnehmung. Diese Studierenden kritisieren die zeitlich versetzte Arbeitsweise, die von anderen Teilnehmenden als positiv empfunden wird. Hier werden lange Wartezeiten und die Ungleichzeitigkeit als Kritikpunkte genannt, da es außerhalb des Chats kaum Möglichkeiten gebe, andere Spieler*innen zu kontaktieren. Die Aussagen mit einer gemischten Bewertung beziehen sich auf diesen genannten Trade-off: Auf der einen Seite wird hervorgehoben, dass das digitale Arbeiten positiv ist, da es autonomer und flexibler wahrgenommen wird. Auf der anderen Seite wird die Verzögerung durch andere Teilnehmende negativ bewertet. Besonders negativ wird angemerkt, dass Teilnehmende während des Planspiels ihre Teilnahme eingestellt haben, ohne dies mitzuteilen, was man gerade online erst verspätet wahrnimmt. Deshalb kommt insbesondere bei den gemischten Reaktionen der Wunsch nach einem Blended-Learning-Konzept auf, was in dieser Aussage gut zusammengefasst ist:

„Something I found myself repeatedly thinking during the simulation how amazing it would be if this simulation could be combined with a physical setting where students can use this website while also being able to meet each other in person."

Die Wirklichkeitsnähe der Simulation wird als wenig ausgeprägt wahrgenommen (siehe Tab. 2). Viele Teilnehmende geben an, Probleme damit zu haben, die Wirklichkeitsnähe einzuschätzen, da sie selbst keine Erfahrungen mit der EU und Lobbyismus haben.

\subsubsection{Ergebnisse der Befragung zu erworbenen/trainierten Fähigkeiten}

Fähigkeiten, hier vor allem fokussiert auf studien- und berufsrelevante Fähigkeiten, wurden vor allem in den Interviews von den Studierenden angesprochen, aber auch in einigen Selbstreflexionen. Es wurde nicht explizit danach gefragt, sondern die Studierenden berichteten selbstständig über positive und einige gemischte Erfahrungen. Die Studierenden mit gemischten Reaktionen sind sich unsicher, ob sich ihre Fähigkeiten verbessert haben, wohingegen die Aussagen aus der Unterkategorie „Positive Bewertung“ sehr klar von einem Erlernen einer Fähigkeit ausgehen. 


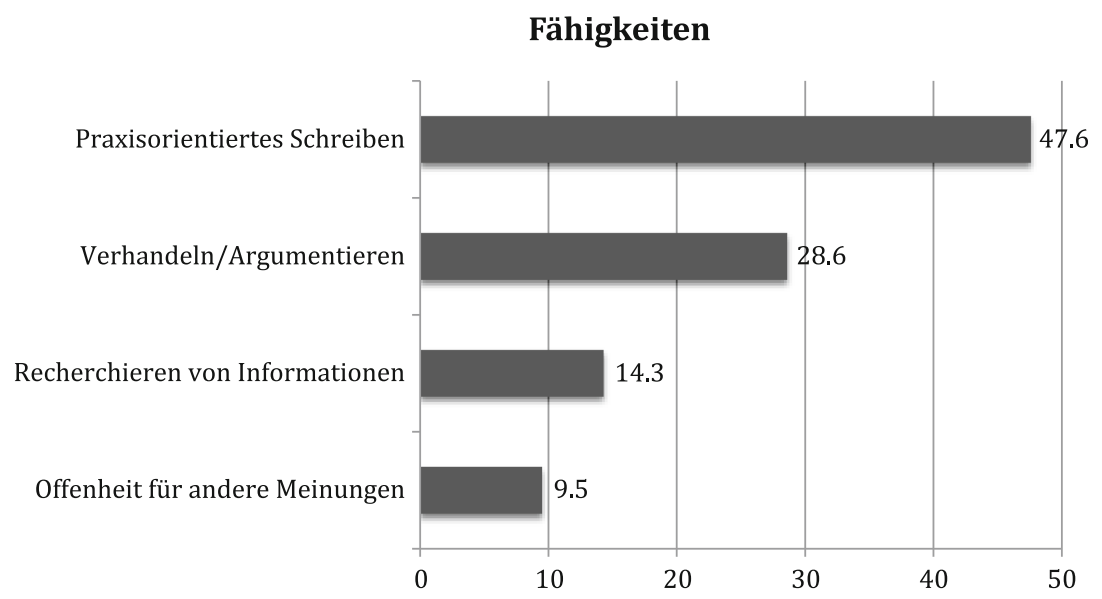

Abb. 1 Ergebnisse der qualitativen Inhaltsanalyse zu den erworbenen studien- und berufsrelevanten Fähigkeiten in Prozent. (Ivens und Kaiser)

Eine Besonderheit der Untersuchung liegt darin, dass anhand der Aussagen der Teilnehmenden induktiv herausgearbeitet wurde, welche Fähigkeiten die Teilnehmenden aus ihrer Sicht erlernen, da vorher keine Zielkriterien dafür festgelegt worden waren. Hierbei wurden alle Fähigkeiten, welche für die Studierenden berufs- und studienrelevant waren, berücksichtigt.

Das digitale Planspiel hilft den Teilnehmenden vor allem beim praxisorientierten Schreiben, zum Beispiel von Policy-Papieren, Presseerklärungen und Stakeholder- Analysen, fast die Hälfte aller Aussagen bezieht sich auf diese Fähigkeit (siehe Abb. 1). Fast $30 \%$ der Aussagen zu Skills beschreiben eine Förderung der kommunikativen Fähigkeiten, wie Argumentieren und Verhandeln. Zusätzlich werden das schnelle Recherchieren von Informationen und die persönliche Offenheit für andere Meinungen und Standpunkte gefördert.

\section{$4 \quad$ Lessons Learned}

\subsection{Learning 1: Das richtige Timing finden}

Das Online-Planspiel hat in der Mitte des deutschen Semesters stattgefunden, da dort keine Prüfungen anstehen und es sich gut mit den Semesterzeiten der 
Partneruniversitäten vereinbaren lässt. In dieser Durchführung ist es zum Teil zu Ausfällen von Studierenden der Universität Antwerpen gekommen, da diese an einer zeitlich überlappenden Face-to-Face-Simulation teilnahmen. Die zeitliche Planung spielt also eine wichtige Rolle für den erfolgreichen Einsatz eines Online-Planspiels. Die meisten Studierenden, die auf den Zeitpunkt des Planspiels eingegangen sind, schlagen vor, es in die vorlesungsfreie Zeit zu verschieben, ein Drittel hätte es gerne direkt zum Anfang des Semesters.

Insgesamt bleibt es schwierig, den perfekten Zeitpunkt für ein internationales Online-Planspiel zu finden, da man die unterschiedlichen Semesterzeiten der Universitäten, die Verfügbarkeiten der Dozierenden und die Semesterpläne der Studierenden miteinbeziehen sollte. Eine frühzeitige Absprache mit allen Beteiligten inklusive der Studierenden bietet sich hier an, damit zumindest arbeitsintensive Zeitintervalle wie Klausurphasen umgangen werden können.

\subsection{Learning 2: Drop-outs verhindern}

Die unvorhersehbare Inaktivität der Teilnehmenden stellt sowohl die Spielenden als auch die Dozierenden vor eine große Herausforderung. Dies bezieht sich auf die sieben Drop-outs, die sich nicht bei den Dozierenden vom Spiel abgemeldet haben. Inaktivität oder ein Drop-out sind im Spiel nur verzögert festzustellen, da die digitale Arbeitsumgebung den Teilnehmenden Autonomie und Flexibilität bietet und diese nicht zwangsweise jeden Tag aktiv sein müssen. Dass die Spielmechanik auf eine wechselseitige Abhängigkeit und hohe Verbindlichkeit zwischen den Aktivitäten der Teilnehmenden setzt, wurde an vielen Stellen in der Inhaltsanalyse deutlich: Viele Studierende haben dieses Verhalten negativ bewertet. Dies äußerte sich insbesondere negativ in der Bewertung des Planspiels und der Wahrnehmung der digitalen Arbeitsumgebung. Durch den Drop-out verzögerte sich der Spielablauf für die anderen Teilnehmenden und einige Studierende wurden demotiviert, da sie sich alleingelassen gefühlt haben. Hervorzuheben ist, dass die Drop-out-Rate unter den Teilnehmenden deutlich geringer ist, die Creditpoints für die Teilnahme und die Erfüllung der Aufgaben bekommen haben. Insofern ist die Vergabe von Creditpoints sehr ratsam. Darüber hinaus könnten obligatorische Motivationsschreiben eingefordert werden, um sich für die Teilnahme zu qualifizieren. 


\subsection{Learning 3: Workload kommunizieren}

Neben den Problemen mit Drop-outs war auch der unterschätzte Workload ein wichtiger Aspekt in der Literatur zu Online-Bildungsangeboten. Insgesamt war die Arbeitsbelastung der Studierenden, die durchgehend am Planspiel teilgenommen haben, höher als die vorher geplanten 60 Minuten pro Tag. Dieser Aspekt konnte auch durch die Inhaltsanalyse bestätigt werden. Die Studierenden empfanden die Arbeitsbelastung als zu hoch, auch in Bezug auf die erhaltenden Creditpoints im Vergleich zu anderen Seminaren. Darauf kann reagiert werden, indem man Aufgaben aus dem Planspiel streicht, die Dauer des Spiels erweitert, das Online-Planspiel ausschließlich in arbeitsintensiveren Modulen anbietet oder die Creditpoints der Arbeitsbelastung anpasst.

\subsection{Learning 4: Face-to-Face-Vorteile nicht vergessen}

Einige Studierende kritisierten, dass ihnen die Face-to-Face-Interaktionen mit anderen Mitspielenden fehlten. Andere haben einen Mix aus Online- und OfflineInteraktionen für das Planspiel vorgeschlagen. Da dies ein internationales Seminar war, lassen sich echte Face-to-Face-Phasen nicht ohne Reiseaufwand ermöglichen. Face-to-Face kann aber ebenso in Videokonferenzen erreicht werden. Es gilt, diese Momente sinnvoll in die bestehende Spielstruktur einzubetten. Der direkte menschliche Kontakt (Sprechen, Hören, Sehen) ist also auch in Online-Angeboten nicht $\mathrm{zu}$ vernachlässigen.

\section{$5 \quad$ Reflexion und Ausblick}

Ist das Online-Planspiel ein gutes Beispiel für Hochschullehre im digitalen Zeitalter? Kann es das Versprechen eines internationalen, digitalen und interaktiven Formates erfüllen?

Die Ergebnisse der exemplarischen Pilotstudie sind grundsätzlich als positiv zu bewerten. Die Inhaltsanalyse weist das Online-Planspiel als ein positives Lernformat aus, sowohl der Lerneffekt, das Erlernen und Verbessern von Fähigkeiten als auch die Internationalisierung durch das Online-Planspiel nehmen fast alle Teilnehmenden ebenfalls als positiv wahr. Gemischt wurde die digitale Arbeitsumgebung aufgefasst. Hier bestätigt sich die bisherige Forschung: Die Autonomie und Flexibilität des Online-Lehrformats werden einerseits geschätzt, andererseits vermissen einige Studierende den Face-to-Face-Kontakt (Schnurr 
et al. 2013). Außerdem wurde der Arbeitsaufwand von den Lehrenden unterschätzt und von den Studierenden dementsprechend als zu hoch empfunden. Dieses Ergebnis deckte sich mit den Forschungen von Vanslambrouck et al. (2018). Zum anderen gab es eine erhöhte Drop-out-Rate, welche sich negativ auf das Spielerlebnis im Online-Planspiel ausgewirkt hat (Cho und Jnassen 2009; Fryer und Bovee 2016).

Allerdings muss auch auf die Limitationen der Studie und das kleine Sample hingewiesen werden. Es gibt einen Selection Bias (Heckmann 1979), da ausschließlich Studierende an den Selbstreflexionen und Interviews teilgenommen haben, welche das Planspiel bis zum Ende mitgespielt haben. Zusätzlich kann bei dieser Evaluation auch ein Upward Bias vorliegen, da Studierende in Evaluationen von Hochschullehre dazu neigen, diese positiver zu bewerten (Goos und Salomons 2017).

Es haben sich einige wichtige Lessons Learned für die Durchführung von Online-Planspielen in der politikwissenschaftlichen Hochschullehre ergeben.

- Um Drop-outs zu verringern, sollten alle am Online-Planspiel beteiligten Studierenden möglichst Creditpoints für die Bearbeitung der Aufgaben erhalten. Auch sollte der Durchführungszeitpunkt genau mit beteiligten Partneruniversitäten abgesprochen werden.

- Der Arbeitsaufwand für ein Online-Planspiel sollte angemessen im Curriculum wiedergegeben werden, sei es durch die Vergabe von Creditpoints oder durch die Einbettung in arbeitsintensive Module.

- Die Einbeziehung von Face-to-Face-Elementen, zum Beispiel durch Videochats, sollte in Online-Planspiele integriert werden.

Die Lessons Learned können hilfreich sein bei der Gestaltung zukünftiger Seminare mit Online-Planspielen. Das exemplarisch untersuchte Online-Planspiel wird aufgrund der Ergebnisse zum Beispiel in einem wesentlich arbeitsintensiveren Modul angeboten, an der Partneruniversität Antwerpen wird die Teilnahme an dem Planspielseminar nun ebenfalls mit Creditpoints bewertet. Videochats zwischen den Akteur*innen während der Durchführung werden empfohlen.

Die zukünftige Anwendung dieses Planspiels wird begleitend beforscht. Damit kann die kleine Samplegröße, eine Limitation dieser Untersuchung, vergrößert werden. Aus Forschungssicht bieten sich empirische Interventionsstudien mit Prä-, Post- und Follow-up-Erhebungen an. Auch Vergleichsstudien zu analogen Seminaren über Lobbyismus im Mehrebenensystem der EU werden empfohlen. 
Die positiven Bewertungen der Studierenden und die inhaltlich flexible Form des Planspiels legen eine Anwendung auch für weitere Fächer sowie fächerübergreifende und internationale Kooperationen zwischen Universitäten nahe. Insgesamt bieten sich sehr viele Anwendungsmöglichkeiten von Online-Planspielen in der Hochschullehre an, um diese digitaler, kooperativer und internationaler $\mathrm{zu}$ gestalten, nicht nur für die Politikwissenschaft. Dieser Beitrag soll deshalb nicht nur weitere Impulse für die wissenschaftliche Debatte über Online-Planspiele setzen, sondern auch Lehrenden als Anregung für die Anwendungsmöglichkeiten in ihren eigenen Fächern und der eigenen Lehre dienen.

\section{Literatur}

Bachen, C. M., Hernández-Ramos, P. F., Raphael, C., \& Waldron, A. (2015). Civic play and civic gaps: Can life simulation games advance educational equity? Journal of Information Technology \& Politics, 12, 378-395. https://doi.org/10.1080/19331681.2015.1101038.

Chernikova, O., Heitzmann, N., Stadler, M., Holzberger, D., Seidel, T., \& Fischer, F. (2020). Simulation-based learning in higher education: A meta-analysis. Review of Educational Research, 90(4), 499-541. https://doi.org/10.3102/0034654320933544.

Cho, M. H., \& Jonassen, D. (2009). Development of the human interaction dimension of the self-regulated learning questionaire in asynchronous online learning environments. Educational Psychology, 29(1), 117-138. https://doi.org/10.1080/01443410802516934.

Deschacht, N., \& Goeman, K. (2015). The effect of blended-learning on course persistence and performance of adult learners: A difference-in-differences analysis. Computers \& Education, 87, 83-89. https://doi.org/10.1016/j.compedu.2015.03.020.

Digitaler Appendix unter: https://bit.ly/31STDwE. Zugegriffen: 26. Okt. 2020.

Duchatelet, D. (2019). „Yes I can! Can I? “ At the heart of self efficacy for negotiating in role play simulations of political decision-making. Antwerpen: University of Antwerp.

Fink, S. (2015). 36 different chocolate directives: How does the setting influence negotiation outcomes in an EU simulation? European Political Science, 14, 241-253. https://doi.org/ 10.1057/eps.2015.21.

Fryer, L., \& Bovee, N. (2016). Supporting students' motivation for e-learning: Teachers matter on- and offline. Internet and Higher Education, 30, 21-29. https://doi.org/10.1016/j.ihe duc.2016.03.003.

Gabriel, S. (2012). Spielend lernen?! Einsatz von digitalen Spielen im Unterricht. In E. Blaschitz, G. Brandhofer, C. Nosko, \& G. Schwed (Hrsg.), Zukunft des Lernens. Wie digitale Medien, Aus- und Weiterbildung verändern (S. 275-290). Glückstadt: Hülsbüsch.

Goos, M., \& Salomons, A. (2017). Measuring teaching quality in higher education: Assessing selection bias in course evaluations. Research in Higher Education, 58, 341-364. https:// doi.org/10.1007/s11162-016-9429-8.

Heckman, J. J. (1979). Sample selection bias as a specification error. Econometrica, 47(1), 153-161. https://doi.org/10.2307/1912352. 
Horn, L., Rubin, O., \& Schouenborg, L. (2016). Undead pedagogy: How a zombie simulation can contribute to teaching international relations. International Studies Perspectives, 17(2), 187-201. https://doi.org/10.1111/insp.12103.

Ivens, S., Wiese, G., Dittert, K., Musshoff, O., \& Oberle, M. (2020). Bringing policy decisions to the people - Education for sustainable development through a digital simulation game. Sustainability, 2(20), 8743. https://doi.org/10.3390/su12208743.

Jones, R., \& Bursens, P. (2018). Die Effekte von aktivierenden Lernumgebungen: Wie Simulationen affektives Lernen fördern. In W. Muno, A. Niemann, \& P. Guasti (Hrsg.), Europa spielerisch lernen. Überlegungen und Praxisbeispiele zu EU-Simulationen (S. 37-51). Wiesbaden: Springer. https://doi.org/10.1007/978-3-658-17463-7_3.

Kaiser, K., Raiser, S., \& Warkalla, B. (2017). Einfach digital? Onlineplanspiele in der politischen Bildung. In A. Petrik \& S. Rappenglück (Hrsg.), Handbuch Planspiele in der politischen Bildung (S. 267-275). Frankfurt a. M.: Wochenschau.

Kuckartz, U. (2018). Qualitative Inhaltsanalyse: Methoden, Praxis, Computerunterstützung (4., überarbeitete Aufl.). Grundlagentexte Methoden. Weinheim: Beltz.

Le, S., Weber, P., \& Ebner, M. (2013). Game-Based Learning. Spielend Lernen? In M. Ebner \& S. Schön (Hrsg.), Lehrbuch für Lernen und Lehren mit Technologien, L3T. (S. 267). Berlin: epubli.

Lohmann, J. R. (2019). Simulations Matter - Wirkungsweisen und Mehrwert von Politiksimulationen. Passau: Universität Passau.

Lybeck, K., Bruhn, D., \& Feyissa, S. (2010). The reality of virtual reality: Second life as a tool for online peer-teaching activities. In M. S. Raisinghani (Hrsg.), Curriculum, learning, and teaching advancements in online education (S. 74-93). Hershey: IGI Global. https:// doi.org/10.4018/978-1-4666-2949-3.ch006.

Meßner, M. T., Schedelik, M., \& Engartner, T. (Hrsg.). (2018). Handbuch Planspiele in der sozialwissenschaftlichen Hochschullehre. Schwalbach/Ts.: Wochenschau.

Muno, W. (2020). Planspiele und Politiksimulationen in der Hochschullehre. Schwalbach/Ts.: Wochenschau.

Naidu, S., Ip, A., \& Linser, R. (2000). Dynamic goal-based role-play simulation on the web: A case study. Journal of Educational Technology \& Society, 3(3), 190-202.

Petrik, A., \& Rappenglück, S. (Hrsg.). (2017). Handbuch Planspiele in der politischen Bildung. Schwalbach/Ts.: Wochenschau.

Raiser, S., Warkalla, B., Schneider, A., \& Kaiser, K. (2018). 'Will It Blend?' Combining online and on-site elements in simulation games. In P. Bursens, V. Donche, D. Gijbels, \& P. Spooren (Hrsg.), Simulations of decision-making as active learning tools. Design and effects of political science simulations (S. 77-92). Cham: Springer International. https:// doi.org/10.1007/978-3-319-74147-5_7.

Schnurr, M. A., De Santo, E., \& Craig, R. (2013). Using a blended-learning approach to simulate the negotiation of a multilateral environmental agreement. International Studies Perspectives, 14(2), 109-120. https://doi.org/10.1111/j.1528-3585.2012.00470.x.

Snyder, K. (2003). Ropes, poles, and space: Active learning in business education. Active Learning in Higher Education, 4(2), 159-167. https://doi.org/10.1177/146978740300400 2004. 
Vanslambrouck, S., Zhu, C., Lombaerts, K., Philipsen, B., \& Tondeur, J. (2018). Students' motivation and subjective task value of participating in online and blended-learning environments. The Internet and Higher Education, 36, 33-40. https://doi.org/10.1016/j.ihe duc.2017.09.002.

Vanslambrouck, S., Zhu, C., Tondeur, J., \& Lombaerts, K. (2016). Adult learners' motivation to participate and perception of online and blended environments. In J. Novotná \& A. Jancarík (Hrsg.), Proceedings of the 15th European conference on e-learning (S. 750-757). Prag: Academic Conference and Publishing International Limited.

Vlachopoulos, D., \& Makri, A. (2017). The effect of games and simulations on higher education: A systematic literature review. International Journal of Educational Technology in Higher Education, 14, 22. https://doi.org/10.1186/s41239-017-0062-1.

Wills, S., Leigh, E., \& Ip, A. (2011). The power of role-based e-learning: Designing and moderating online role play (1. Aufl.). New York: Routledge.

Open Access Dieses Kapitel wird unter der Creative Commons Namensnennung 4.0 International Lizenz (http://creativecommons.org/licenses/by/4.0/deed.de) veröffentlicht, welche die Nutzung, Vervielfältigung, Bearbeitung, Verbreitung und Wiedergabe in jeglichem Medium und Format erlaubt, sofern Sie den/die ursprünglichen Autor(en) und die Quelle ordnungsgemäß nennen, einen Link zur Creative Commons Lizenz beifügen und angeben, ob Änderungen vorgenommen wurden.

Die in diesem Kapitel enthaltenen Bilder und sonstiges Drittmaterial unterliegen ebenfalls der genannten Creative Commons Lizenz, sofern sich aus der Abbildungslegende nichts anderes ergibt. Sofern das betreffende Material nicht unter der genannten Creative Commons Lizenz steht und die betreffende Handlung nicht nach gesetzlichen Vorschriften erlaubt ist, ist für die oben aufgeführten Weiterverwendungen des Materials die Einwilligung des jeweiligen Rechteinhabers einzuholen.

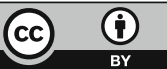

